\title{
Patterns of Diabetes Care Among People with Type 1 Diabetes During Ramadan: An International Prospective Study (DAR-MENA T1DM)
}

\author{
Fatheya F. Al Awadi (D) - Akram Echtay · Monira Al Arouj · \\ Sobia Sabir Ali · Naim Shehadeh • Abdulrahman Al Shaikh • \\ Khier Djaballah · Cecile Dessapt-Baradez • Mohamad Omar Abu-Hijleh • \\ Abdullah Bennakhi · Mohamed El Hassan Gharbi · Khaled El Sayed El Hadidy • \\ Faris Abdul Kareem Khazaal · Mohamed M. Hassanein
}

Received: December 17, 2019 / Published online: March 6, 2020

(C) The Author(s) 2020

\section{ABSTRACT}

Introduction: To describe the characteristics and care of participants with type 1 diabetes during Ramadan in the Middle East and North Africa.

Methods: The DAR-MENA (Diabetes and Ramadan-Middle East and North Africa) study was a prospective, observational study of adults

Enhanced Digital Features To view enhanced digital features for this article go to https://doi.org/10.6084/ m9.figshare.11860932.

Electronic Supplementary Material The online version of this article (https://doi.org/10.1007/s12325020-01267-4) contains supplementary material, which is available to authorized users.

F. F. Al Awadi ( $₫)$ · M. M. Hassanein

Dubai Hospital, Dubai, United Arab Emirates

e-mail: ffAlawadi@dha.gov.ae

A. Echtay

Rafic Hariri University Hospital, Beirut, Lebanon

M. Al Arouj · A. Bennakhi

Dasman Diabetes Institute, Kuwait City, Kuwait

S. Sabir Ali

Lady Reading Hospital, Peshawar, Pakistan

N. Shehadeh

Rambam Medical Center, Haifa, Israel

A. Al Shaikh

King Abdulaziz University Hospital, Jeddah, Saudi Arabia with type 1 and type 2 diabetes who were Muslim and did/did not intend to fast during Ramadan 2016. Baseline data were collected 6 weeks prior to Ramadan, with a follow-up visit 1-2 months after Ramadan. This is the analysis of the population with type 1 diabetes. Measurements included proportion who fasted, reasons for fasting and not fasting, changes in diabetes treatment, hypoglycemic events, and proportion with access to diabetes education.

Results: Of 136 participants with type 1 diabetes, $76.9 \%(100 / 130)$ fasted for at least 1 day, $72.3 \%$ (94/130) fasted for at least 15 days, and $48.5 \%$ (63/130) fasted for 30 days. The majority $(63.0 \%$, $63 / 100$ ) reported personal decision as a reason to fast. Fear of diabetic complications $(58.6 \%, 17 / 29)$ and previous complications related to fasting

K. Djaballah

Sanofi, Paris, France

C. Dessapt-Baradez

Sanofi, Guildford, UK

M. Omar Abu-Hijleh

Jordan Hospital, Amman, Jordan

M. El Hassan Gharbi

Ibn Sina Hospital, Rabat, Morocco

K. El Sayed El Hadidy

Beni Suef University, Beni Suef, Egypt

F. Abdul Kareem Khazaal

Alkindy Obesity Center, Baghdad, Iraq 
$(48.3 \%, 14 / 29)$ were the most common reasons for not fasting. Adjustment of diabetic medication regimen occurred for $84.6 \%(115 / 136)$ of participants, and $72.8 \%(99 / 136)$ changed their treatment dose. The incidence and number of adverse events for confirmed and severe hypoglycemia were similar before and during Ramadan. Almost half of participants had access to diabetes education $(45.6 \%, 62 / 136)$.

Conclusion: The DAR-MENA study showed that despite the risks associated with fasting for people with type 1 diabetes, almost half fasted for the full 30 days of Ramadan with no significant change in hypoglycemia events. Since the current International Diabetes Federation and Diabetes and Ramadan guidelines do not endorse fasting for people with type 1 diabetes, it is important that those who insist on fasting work closely with their healthcare practitioner to avoid any complications.

Keywords: Epidemiology; Hypoglycemia; Ramadan; Type 1 diabetes

\section{Key Summary Points}

Why carry out this study?

Despite limited research on fasting during Ramadan in people with type 1 diabetes, and guideline recommendations against fasting due to the potential of a high risk of complications, many people with type 1 diabetes still fast during Ramadan.

This observational study was conducted in the Middle Eastern and North African (MENA) region to provide an epidemiologic analysis of the characteristics and patterns of care of people with type 1 diabetes during Ramadan.

\section{What was learned from the study?}

The key finding of this study was that despite the risks associated with fasting for people with type 1 diabetes, almost half fasted for Ramadan (full 30 days) and they experienced a significant improvement in glycemic control following Ramadan, with no significant change in hypoglycemia events.
The results of this study provide new insights into the characteristics and pattern of fasting and care for people with type 1 diabetes before and during Ramadan, and may help to ensure the safety of people with type 1 diabetes who choose to fast during Ramadan.

In particular, the results indicate that people with type 1 diabetes who insist on fasting need to work closely with their healthcare practitioner to ensure that they reduce their risk of complications and highlight the importance of Ramadanfocused education for patients.

\section{INTRODUCTION}

During the lunar-based month of Ramadan, Muslims fast from predawn to sunset. The length of the fast each day depends on the geographic location and season, and may range from a few hours to more than $20 \mathrm{~h}$. Fasting during Ramadan is compulsory for all healthy adult Muslims, although those with medical conditions, including many with diabetes, are exempted from fasting according to the Qur'an [1]. During this holy month, there are also changes to daily activities and shorter sleeping hours.

For people with type 1 diabetes who fast during Ramadan, insulin deficiency can lead to excessive glycogen breakdown, increased gluconeogenesis, and ketogenesis [2, 3]. This results in a very high risk of complications, including hypoglycemia, hyperglycemia, diabetic ketoacidosis, dehydration, and thrombosis [2-4]. Compared with type 2 diabetes, the risk of hypoglycemia is higher with type 1 diabetes $[3,5]$. As a result of these risks, the International Diabetes Federation and Diabetes and Ramadan (IDF-DAR) Practical Guidelines recommend that people with type 1 diabetes, even if it is well controlled, should not fast [3]; those with a history of recurrent hypoglycemia, hypoglycemia unawareness, poor diabetes control, brittle diabetes, non-compliance with medical 
treatment, or who are unwilling or unable to monitor their blood glucose levels are considered at very high risk of complications and must not fast [3]. Large-scale studies specific to fasting during Ramadan for type 1 diabetes are limited. One previous study (EPIDIAR [Epidemiology of Diabetes and Ramadan]) demonstrated that about $43 \%$ of people with type 1 diabetes fasted for at least 15 days during Ramadan compared with $79 \%$ of individuals with type 2 diabetes [5]. In the DAR-MENA (Diabetes and RamadanMiddle East and North Africa) type 2 diabetes mellitus (T2DM) study, $86 \%$ of participants fasted for at least 15 days [6].

Fasting during Ramadan has been associated with significant and variable changes in the attitudes and behaviors of people with type 1 diabetes [7]. In one study of people with type 1 diabetes, the timing of the main meal at sunset (Iftar) differed widely between individuals. Furthermore, a large number of people did not perform regular self-monitoring of blood glucose during fasting, particularly those on multiple daily insulin injections. Studies have shown that it is possible for people with type 1 diabetes to participate safely in prolonged fasts if they work with their healthcare practitioner and receive education to manage their condition effectively; however, these studies were performed with small numbers of participants $[8,9]$. A pre-Ramadan medical assessment is essential, and people with diabetes should be educated on the risks associated with fasting and techniques to decrease these risks [3]. Modifications to insulin therapy and/or dosing regimen for the fasting period are necessary [3]. Human insulin therapy should be switched to insulin analogues, if possible, and insulin dose titration should be performed every 3 days according to blood glucose levels [3].

DAR-MENA was an observational study, which was conducted in the Middle Eastern and North African (MENA) region to provide an epidemiologic analysis of the characteristics and patterns of care of people with type 1 or type 2 diabetes during Ramadan [6]. This paper reports the findings on daily fasting practices during Ramadan for people with type 1 diabetes by capturing data before and during the holy month, including pattern of care (particularly medications), changes of insulin regimen and dosage, glycemic control, hypoglycemia risk, lifestyle changes, and patient education on diabetes management.

\section{METHODS}

\section{Study Design}

The study methodology of DAR-MENA and the results from the type 2 diabetes subpopulation have been published [6]. Briefly, DAR-MENA was an international, multicenter, prospective, observational study in the MENA region for Ramadan 2016 (fasting period: dawn of June 6 to evening of July 5) with a target enrollment of 2000 participants with type 1 or type 2 diabetes. In the overall study, the inclusion criteria comprised adult participants who were aged at least 18 years with type 1 or type 2 diabetes, and who were Muslim and did or did not intend to fast. Baseline data were collected in the 6 weeks prior to Ramadan, with a follow-up visit 1-2 months after Ramadan. The results shown here focus on the type 1 diabetes population.

The manuscript followed the STrengthening the Reporting of OBservational studies in Epidemiology (STROBE) guidelines [10]. DARMENA was conducted in accordance with the ethical principles laid down in the Declaration of Helsinki and complied with all international guidelines, as well as national laws and regulations of each country in which the registry study was performed. The study protocol was approved by the ethics committee of each participating center (please see Table $\$ 9$ in the supplementary material for ethics committee names). Participants signed a consent form to participate in the study. Please note that this study was not added to a clinical trial registry as it was a non-interventional, prospective, observational study that did not meet the definition for clinical trial registration as specified by the World Health Organization.

\section{Measurements}

Variables measured or recorded were as follows: mean number of days fasted; number of 
participants who fasted for at least 1 day, at least 15 days, and 30 days; and reasons for fasting and not fasting, as well as diabetes treatment among participants who fasted, including any modifications in antidiabetes medication or doses of medication. Change in glycemic profile (glycated hemoglobin [HbA1c], fasting plasma glucose [FPG], and postprandial plasma glucose [PPG]), lipid profiles (total cholesterol, triglycerides, high-density lipoprotein, and low-density lipoprotein), and biometric measures (weight, systolic/diastolic blood pressure, waist circumference, and heart rate) was determined between the period before Ramadan and during Ramadan. Values up to 6 months before baseline were used to determine baseline data for the glycemic and lipid profiles. Changes in lifestyle and access to education on diabetes management were also recorded.

Incidence and number of adverse events (AEs) were recorded for symptomatic confirmed hypoglycemia, severe hypoglycemia, symptomatic confirmed hyperglycemia, and severe hyperglycemia during the 1 month (4 weeks before visit 1) before Ramadan and during the Ramadan fasting period. Symptomatic confirmed hypoglycemia was defined as an event with clinical symptoms that are considered to result from hypoglycemia and was confirmed by a self-measured plasma glucose (SMPG) level of less than $70 \mathrm{mg} / \mathrm{dL}$. Severe hypoglycemia was defined as a hypoglycemia event requiring assistance due to acute neurologic impairment directly resulting from hypoglycemia. Severe episodes of hypoglycemia were self-reported by participants, and participants were also asked by study investigators if they had experienced any hypoglycemic episodes that required hospitalization. Symptomatic confirmed hyperglycemia was defined as an event with clinical symptoms that are considered to result from hyperglycemia and was confirmed by an SMPG level of at least $200 \mathrm{mg} / \mathrm{dL}$.

\section{Statistical Analysis}

For biochemical and biometric measures, the paired $t$ test was used to compare between metric variables, the McNemar-Bowker test was used to compare between physical activity data, and the McNemar test was used to compare between categorical variables before and after Ramadan. For comparisons of hypoglycemia/ hyperglycemia data between the 4 weeks before Ramadan and during Ramadan, the McNemar test was used for paired categorical comparison and the paired $t$ test was used for paired numerical comparisons.

Descriptive quantitative variables were reported with mean and standard deviation (SD), and descriptive categorical data were reported by number and percentage of the study population.

As not all data were available for all measurements, the total $n$ values vary between measurements. Imputation of missing data was not performed.

\section{RESULTS}

\section{Population Demographics and Baseline Clinical Characteristics}

Participant disposition data for the DAR-MENA study have been published [6]. In brief, a total of 1894 participants with type 1 and type 2 diabetes from the MENA region were enrolled in DAR-MENA, of whom 1885 were evaluable. Of these eligible participants, 136 had type 1 diabetes and were included in this present analysis.

People with type 1 diabetes had a mean age of 32.0 years, had a disease duration of 14.0 years, and $55.1 \%$ were male (Table 1 ). At baseline, the mean HbA1c was $8.3 \%$ $(67 \mathrm{mmol} / \mathrm{mol})$. The number of comorbidities at baseline was low; the most common were hyperlipidemia, hypertension, and hypothyroidism ( $n=3$ for each) (Table $\mathrm{S} 1$ in the supplementary material). All participants were taking insulin. There were $87(64.0 \%)$ participants receiving rapid-acting insulin, 10 (7.4\%) receiving intermediate-acting insulin, 67 (49.3\%) receiving basal insulin analogues, and $56(41.2 \%)$ receiving premix insulin (Table S2 in the supplementary material). 
Table 1 Baseline demographics, participant characteristics, lifestyle, and medical history in participants with type 1 diabetes $(n=136)$

\begin{tabular}{|c|c|c|c|}
\hline Characteristic & Total $n$ & $n$ or mean & $\%$ or $\pm S D$ \\
\hline Men & 136 & 75 & 55.1 \\
\hline Age [range] (years) & 136 & $32.0[18.4-66.4]$ & \pm 9.5 \\
\hline Weight (kg) & 136 & 74.8 & \pm 14.3 \\
\hline Waist circumference $(\mathrm{cm})$ & 103 & 89.3 & \pm 16.3 \\
\hline Body mass index $\left(\mathrm{kg} / \mathrm{m}^{2}\right)$ & 136 & 26.3 & \pm 4.5 \\
\hline \multicolumn{4}{|l|}{ Vital signs } \\
\hline SBP (mmHg) & 135 & 122.1 & \pm 11.3 \\
\hline $\mathrm{DBP}(\mathrm{mmHg})$ & 135 & 76.6 & \pm 8.5 \\
\hline Heart rate (beats/min) & 130 & 79.1 & \pm 9.0 \\
\hline \multicolumn{4}{|l|}{ Lifestyle } \\
\hline \multicolumn{4}{|l|}{ Physical activity } \\
\hline Inactive & 135 & 16 & 11.9 \\
\hline Low & 135 & 38 & 28.1 \\
\hline Moderate & 135 & 70 & 51.9 \\
\hline High & 135 & 11 & 8.1 \\
\hline Smoking status (yes) & 135 & 14 & 10.4 \\
\hline \multicolumn{4}{|l|}{ Daily working and sleeping hours } \\
\hline Working (h/day) & 127 & 7.7 & \pm 3.0 \\
\hline Sleeping (h/day) & 135 & 7.8 & \pm 1.2 \\
\hline \multicolumn{4}{|l|}{ Clinical characteristics } \\
\hline HbAlc (\%) & 134 & 8.3 & \pm 1.7 \\
\hline $\mathrm{HbAlc}(\mathrm{mmol} / \mathrm{mol})$ & 134 & 67 & \pm 18 \\
\hline $\mathrm{FPG}(\mathrm{mg} / \mathrm{dL})$ & 128 & 161.4 & \pm 58.9 \\
\hline PPG (mg/dL) & 112 & 214.3 & \pm 66.1 \\
\hline $\mathrm{LDL}(\mathrm{mg} / \mathrm{dL})$ & 74 & 108.2 & \pm 28.0 \\
\hline $\mathrm{HDL}(\mathrm{mg} / \mathrm{dL})$ & 73 & 47.2 & \pm 12.4 \\
\hline Triglycerides $(\mathrm{mg} / \mathrm{dL})$ & 91 & 135.2 & \pm 130.9 \\
\hline Total cholesterol (mg/dL) & 90 & 172.3 & \pm 38.4 \\
\hline Serum creatinine $(\mathrm{mg} / \mathrm{dL})$ & 98 & 1.1 & \pm 1.0 \\
\hline Medical and surgical history (yes) & 136 & 24 & 17.6 \\
\hline Concomitant medications (yes) & 136 & 52 & \pm 38.2 \\
\hline Duration of diabetes (years) & 136 & 14.0 & \pm 7.7 \\
\hline
\end{tabular}


Table 1 continued

\begin{tabular}{llll}
\hline Characteristic & Total $\boldsymbol{n}$ & $\boldsymbol{n}$ or mean & \% or $\mathbf{\pm}$ SD \\
\hline Family history of diabetes (yes) & 135 & 92 & 68.1 \\
Late diabetes complications (yes) ${ }^{\mathrm{a}}$ (most common listed) & 136 & 46 & 33.8 \\
Diabetic neuropathy & 136 & 38 & 27.9 \\
Diabetic retinopathy & 136 & 12 & 8.8 \\
Diabetic nephropathy & 136 & 11 & 8.1 \\
Diabetes management before Ramadan, $n$ (\%) & & & \\
Physical activity only & 136 & 0 & 0 \\
OAD alone & 136 & 0 & 0 \\
OADs & 136 & 9 & 6.6 \\
1 OAD & 9 & 6 & 22.2 \\
2 OADs & 9 & 2 & 11.1 \\
3 OADs & 9 & 1 & 0 \\
$>3$ OADs & 9 & 0 & 100 \\
Injectable therapy & 136 & 136 & 100 \\
Insulin & 136 & 136 & 0 \\
GLP-1 RA & 136 & 0 & 93.4 \\
Injectable alone & 136 & 127 & \\
\hline
\end{tabular}

$D B P$ diastolic blood pressure, FPG fasting plasma glucose, GLP-1 RA glucagon-like peptide 1 receptor agonist, $H b A 1 c$ glycated hemoglobin, $H D L$ high-density lipoprotein, $L D L$ low-density lipoprotein, $O A D$ oral antidiabetes drug, $P P G$ postprandial plasma glucose, $S B P$ systolic blood pressure, $S D$ standard deviation

${ }^{a}$ Late diabetes complications included diabetic neuropathy, diabetic nephropathy, diabetic retinopathy, coronary artery disease, myocardial infarction, peripheral vascular disease, stroke, microalbuminuria, and neuropathic arthropathy

${ }^{b}$ Nine participants were taking OADs in combination with insulin at baseline; however, one of these patients reported that they planned to stop insulin during Ramadan (please see Table S2 in the supplementary material for more details on antidiabetes therapies used at baseline)

\section{Fasting Practice and Pattern of Care Before and During Ramadan}

A total of $76.9 \%(n=100 / 130$ [participants with evaluable data]) of the type 1 diabetes population fasted during Ramadan for at least 1 day, $72.3 \%$ fasted for at least 15 days, and $48.5 \%$ fasted for the full 30 days (Table 33 in the supplementary material). In these participants, the mean $\pm \mathrm{SD}$ duration of fasting was $26.9 \pm 5.9$ days, with a mean \pm SD daily fasting duration of $15.8 \pm 0.9 \mathrm{~h}$ during Ramadan 2016 $(n=100)$.
The majority (63.0\%) of people with type 1 diabetes listed "Personal decision" as a reason to fast (Table S4 in the supplementary material). "To cope with family and community" was given as a reason to fast for $24.0 \%$ of participants. The most common reasons for not fasting were "Fear of diabetic complications" $(60.0 \%)$ and "Previous experience of acute diabetic complication related to fasting" (50.0\%) (Table S4 in the supplementary material). "Other health-related issues (not related to diabetes)" was a reason not to fast for $10.0 \%$ of 

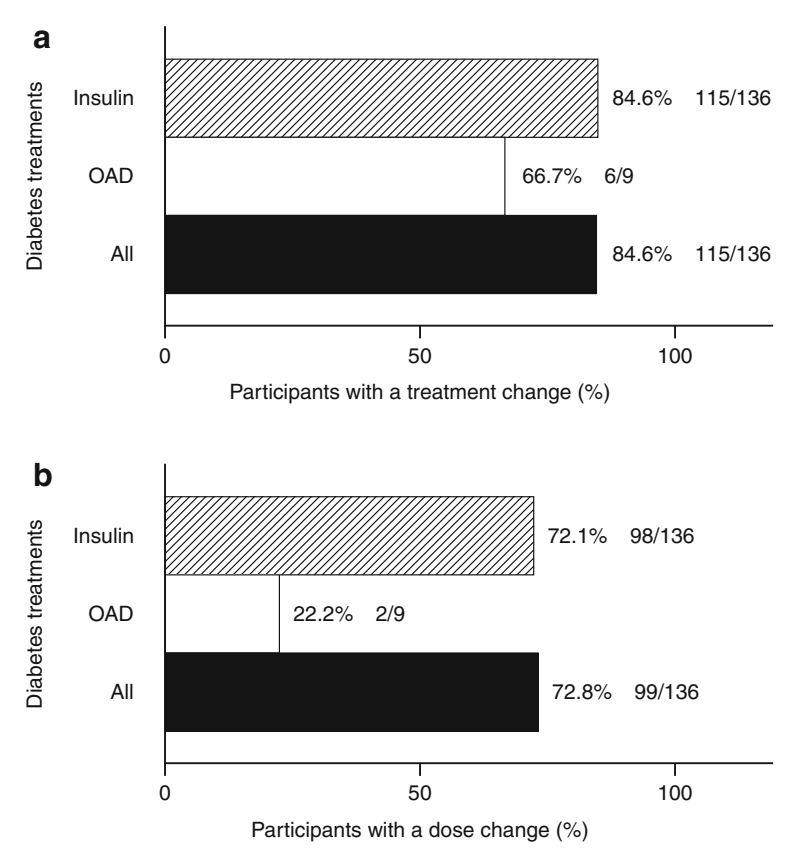

Fig. 1 Modifications in diabetes treatment in participants with type 1 diabetes $(n=136)$ for a change in treatment ${ }^{a}$ and $\mathbf{b}$ change in dose. ${ }^{a}$ Change in treatment is defined as participants who had a change in drug and/or dose. $O A D$ oral antidiabetes drug

participants, and only $6.7 \%$ had been advised not to fast by healthcare professionals.

All participants were receiving insulin at baseline; $84.6 \%$ of participants experienced a change in their diabetic treatment regimen, and $72.1 \%$ experienced modifications in their insulin dose (Fig. 1). Of these participants, $80.6 \%$ (79/98) had one or more of their insulin doses reduced. Of the $27.9 \%$ of participants who did not change their insulin dose, the insulin regimens were as follows: rapid-acting insulin in $60.5 \%(n=23)$, intermediate-acting insulin in $7.9 \%(n=3)$, basal insulin analogues in $47.4 \%$ $(n=18)$, premix insulin in $44.7 \%(n=17)$, and "other" in $2.6 \%$ ( $n=1$; participant was using an insulin pump; insulin type was not specified).

\section{Biochemical and Biometric Measures Before and During Ramadan}

Compared with before Ramadan, there were significant changes in biochemical and biometric measures during Ramadan in some cases
(Table 2). Although change in waist circumference was significant, it was not considered clinically meaningful and there was no significant change in weight. The number of working hours per day decreased significantly by $1.2 \mathrm{~h}$ $(P<0.0001)$, but the change in numbers of hours of sleep was not significant.

The glycemic profile improved during Ramadan compared with before (Table 2). Mean $\mathrm{HbA} 1 \mathrm{c}$ decreased by $0.6 \%(6.2 \mathrm{mmol} / \mathrm{mol})$ to $7.8 \%(62 \mathrm{mmol} / \mathrm{mol}) \quad(P<0.0001)$, mean FPG decreased by $21.2 \mathrm{mg} / \mathrm{dL}$ to $143.2 \mathrm{mg} / \mathrm{dL}$ $(P<0.0001)$, and mean PPG decreased by $22.8 \mathrm{mg} / \mathrm{dL}$ to $191.9 \mathrm{mg} / \mathrm{dL}(P<0.0001)$. Mean lipid profile values did not change significantly during Ramadan, except for total cholesterol, which was increased by $6.3 \mathrm{mg} / \mathrm{dL}(P=0.021)$.

\section{Hypoglycemia and Hyperglycemia Before and During Fasting}

The incidence of confirmed hypoglycemia and number of confirmed hypoglycemia AEs did not change significantly from the 4 weeks before Ramadan ( $n=136 ; 27.2 \%, 0.93$ events/month/ participant) to during Ramadan $(n=130$; 23.1\%, 0.79 events/month/participant) (Fig. 2). During Ramadan, the incidence of severe hypoglycemia and number of AEs were low (Fig. 2). There was no significant change in the incidence or number of AEs for severe hypoglycemia, confirmed hyperglycemia, and severe hyperglycemia during Ramadan compared with before (Fig. 2; Table S5 in the supplementary material).

\section{Lifestyle Changes and Patient Education on Diabetes Management}

Most people (95.4\%) reported lifestyle changes during Ramadan fasting (Table S6 in the supplementary material). Participants reported changes for working hours $(67.5 \%)$, sleep duration $(67.2 \%)$, physical activity (45.0\%), and smoking (7.8\%). Of those reporting a change, working hours decreased in $84.3 \%$, sleep duration decreased in $54.5 \%$, and physical activity decreased in 60.3\%; smoking decreased in all 10 participants who reported on this lifestyle 
Table 2 Comparison between before and after Ramadan for biochemical and biometric measures in participants with type 1 diabetes $(n=136)$

\begin{tabular}{|c|c|c|c|c|c|c|c|c|}
\hline \multirow[t]{2}{*}{ Characteristic } & \multicolumn{3}{|c|}{ Before Ramadan } & \multicolumn{3}{|c|}{ After Ramadan } & \multirow[t]{2}{*}{ Change } & \multirow[t]{2}{*}{$P$ value } \\
\hline & $\begin{array}{l}\text { Total } \\
n\end{array}$ & $\begin{array}{l}\text { Count/ } \\
\text { mean }\end{array}$ & $\begin{array}{l}\% / \\
\pm \text { SD }\end{array}$ & $\begin{array}{l}\text { Total } \\
n\end{array}$ & $\begin{array}{l}\text { Count/ } \\
\text { mean }\end{array}$ & $\begin{array}{l}\% / \\
\pm S D\end{array}$ & & \\
\hline Weight $(\mathrm{kg})$ & 136 & 74.8 & \pm 14.3 & 130 & 75.0 & \pm 14.4 & $0.4 \pm 3.8$ & $0.255^{a}$ \\
\hline $\begin{array}{l}\text { Waist circumference } \\
(\mathrm{cm})\end{array}$ & 103 & 89.3 & \pm 16.3 & 100 & 88.7 & \pm 15.8 & $-1.4 \pm 6.0$ & $0.03^{a}$ \\
\hline $\mathrm{SBP}(\mathrm{mmHg})$ & 135 & 122.1 & \pm 11.3 & 130 & 121.5 & \pm 11.9 & $-0.5 \pm 8.7$ & $0.485^{a}$ \\
\hline $\mathrm{DBP}(\mathrm{mmHg})$ & 135 & 76.6 & \pm 8.5 & 130 & 76.8 & \pm 8.3 & $0.08 \pm 6.3$ & $0.888^{\mathrm{a}}$ \\
\hline Heart rate (beats/min) & 130 & 79.1 & \pm 9.0 & 126 & 78.6 & \pm 6.9 & $-0.6 \pm 7.6$ & $0.360^{\mathrm{a}}$ \\
\hline Working (h/day) & 127 & 7.7 & \pm 3.0 & 124 & 6.4 & \pm 2.8 & $-1.2 \pm 1.9$ & $<0.0001^{\mathrm{a}}$ \\
\hline Sleeping (h/day) & 135 & 7.8 & \pm 1.2 & 129 & 7.9 & \pm 2.0 & $0.1 \pm 1.8$ & $0.574^{a}$ \\
\hline \multicolumn{9}{|l|}{ Physical activity } \\
\hline Inactive & 135 & 16 & 11.9 & 130 & 17 & 13.1 & & $0.406^{\mathrm{b}}$ \\
\hline Low & 135 & 38 & 28.1 & 130 & 45 & 34.6 & & \\
\hline Moderate & 135 & 70 & 51.9 & 130 & 58 & 44.6 & & \\
\hline High & 135 & 11 & 8.1 & 130 & 10 & 7.7 & & \\
\hline \multicolumn{9}{|l|}{ Smoker } \\
\hline Yes & 135 & 14 & 10.4 & 136 & 13 & 9.6 & & $1^{\mathrm{c}}$ \\
\hline No & 135 & 121 & 89.6 & 136 & 123 & 90.4 & & \\
\hline HbAlc (\%) & 134 & 8.3 & \pm 1.7 & 115 & 7.8 & \pm 1.2 & $-0.6 \pm 1.0$ & $<0.0001^{\mathrm{a}}$ \\
\hline $\mathrm{HbAlc}(\mathrm{mmol} / \mathrm{mol})$ & 134 & 67 & \pm 18 & 115 & 62 & \pm 14 & $-6.2 \pm 11.1$ & $<0.0001^{\mathrm{a}}$ \\
\hline $\mathrm{FPG}(\mathrm{mg} / \mathrm{dL})$ & 128 & 161.4 & \pm 58.9 & 121 & 143.2 & \pm 43.4 & $-21.2 \pm 42.2$ & $<0.0001^{\mathrm{a}}$ \\
\hline $\mathrm{PPG}(\mathrm{mg} / \mathrm{dL})$ & 112 & 214.3 & \pm 66.1 & 106 & 191.9 & \pm 53.8 & $-22.8 \pm 58.3$ & $<0.0001^{\mathrm{a}}$ \\
\hline $\mathrm{LDL}(\mathrm{mg} / \mathrm{dL})$ & 74 & 108.2 & \pm 28.0 & 58 & 116.6 & \pm 33.7 & $4.3 \pm 20.2$ & $0.152^{\mathrm{a}}$ \\
\hline $\mathrm{HDL}(\mathrm{mg} / \mathrm{dL})$ & 73 & 47.2 & \pm 12.4 & 58 & 48.7 & \pm 15.0 & $0.4 \pm 9.9$ & $0.777^{\mathrm{a}}$ \\
\hline Triglycerides $(\mathrm{mg} / \mathrm{dL})$ & 91 & 135.2 & \pm 130.9 & 73 & 128.1 & \pm 46.7 & $-2.2 \pm 31.9$ & $0.590^{\mathrm{a}}$ \\
\hline $\begin{array}{l}\text { Total cholesterol (mg/ } \\
\text { dL) }\end{array}$ & 90 & 172.3 & \pm 38.4 & 72 & 181.6 & \pm 39.2 & $6.3 \pm 20.6$ & $0.021^{\mathrm{a}}$ \\
\hline
\end{tabular}

$D B P$ diastolic blood pressure, $F P G$ fasting plasma glucose, $H b A 1 c$ glycated hemoglobin, $H D L$ high-density lipoprotein, $L D L$ low-density lipoprotein, $P P G$ postprandial glucose, $S B P$ systolic blood pressure, $S D$ standard deviation

a Paired $t$ test used to compare between metric variables before and after Ramadan

b McNemar-Bowker test used to compare between physical activity data before and after Ramadan

${ }^{c}$ McNemar test used to compare between categorical variables before and after Ramadan 

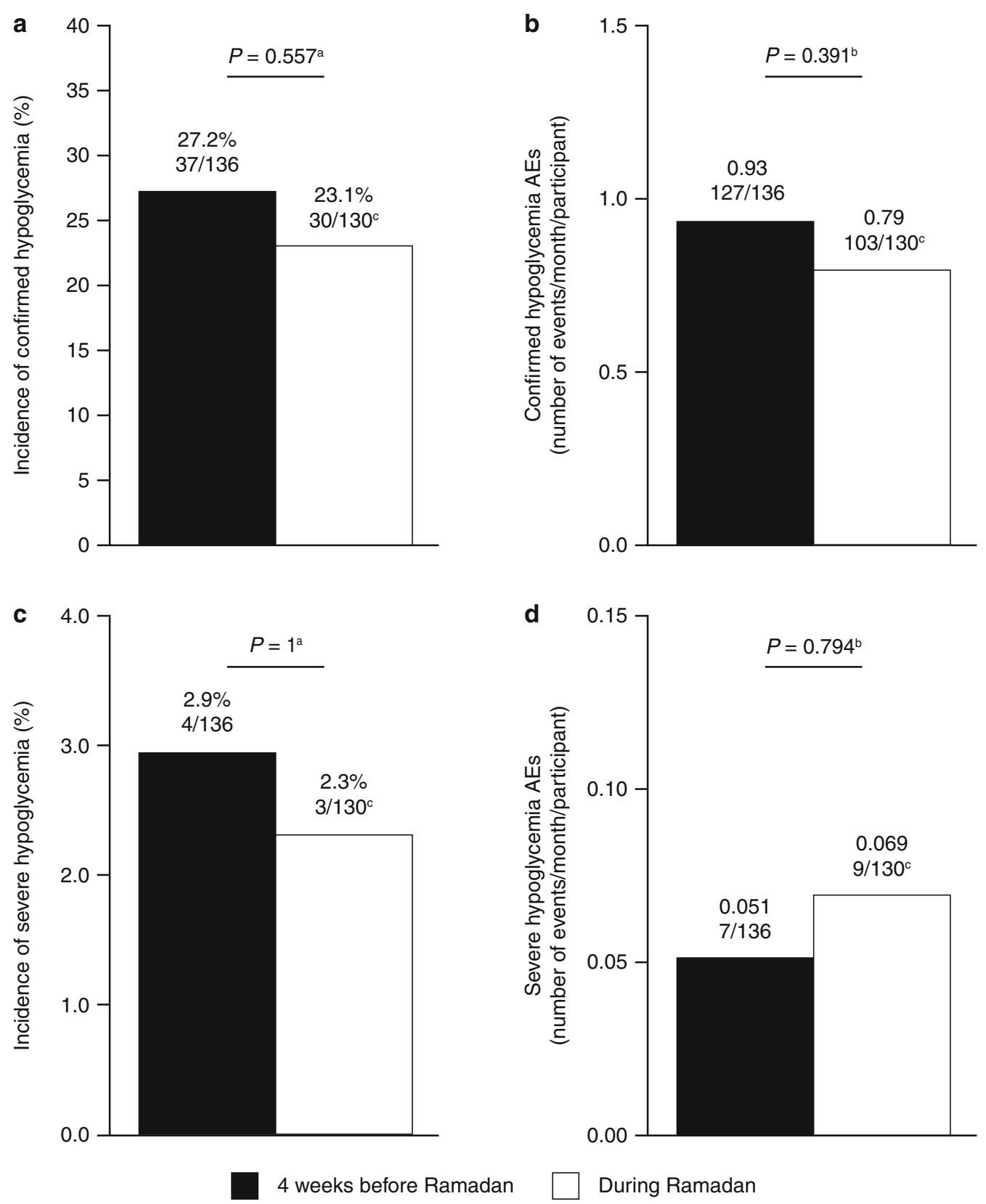

Fig. 2 a Incidence of confirmed hypoglycemia, b confirmed hypoglycemia AEs, c incidence of severe hypoglycemia, and $\mathbf{d}$ confirmed severe hypoglycemia AEs before and during Ramadan in participants with type 1 diabetes

change. Overall food intake during Ramadan was altered in $62.3 \%$ of participants; in those reporting changes, $51.7 \%$ of participants reported a decrease in consumption of carbohydrates, $50.8 \%$ decreased their sugar intake, $92.6 \%$

$(n=136) .{ }^{a} \mathrm{McNemar}$ test was used for paired categorical comparisons. ${ }^{b}$ Paired $t$ test was used for paired numerical comparisons. ${ }^{\text {c }} \mathrm{Six}$ participants with type 1 diabetes were missing hypoglycemia data. $A E$ adverse event

increased the amount of protein that they consumed, $91.8 \%$ increased their vegetable intake, and $60.5 \%$ decreased their consumption of fats. Of the $63.1 \%$ reporting changes in fluid intake, most reported an increase (80.5\%). 
Nearly half of participants with type 1 diabetes had access to diabetes education (45.6\%) (Table S7 in the supplementary material). Of those receiving education, it was accessible through face-to-face diabetes education sessions (45.2\%), diabetes education programs $(25.8 \%)$, diabetes self-education $(27.4 \%)$, and websitebased diabetes education (21.0\%).

\section{DISCUSSION}

DAR-MENA is the first prospective, large study investigating diabetes before and during Ramadan. The type 1 diabetes DAR-MENA analysis provides in-depth information on the characteristics and pattern of care of relatively young and healthy people with type 1 diabetes before and during Ramadan fasting. Importantly, it provides the reasons (outlined below) that prompted people to choose whether to fast during Ramadan. The study found that the majority of people $(72.3 \%)$ fasted for at least 15 days, and nearly half (48.5\%) fasted for the full 30 days of Ramadan without a significant increase in confirmed and severe hypoglycemia events.

The DAR-MENA type 1 diabetes population was young, with a mean age of 32 years and a low number of comorbidities at baseline. The mean duration of type 1 diabetes was 14 years and glycemic control was relatively poor with a mean HbA1c of $8.3 \% \quad(67 \mathrm{mmol} / \mathrm{mol})$. As expected, all participants were taking insulin; 49.3\% were receiving basal insulin analogues.

The role of structured education for patients is well established in the management of diabetes, and guidelines state that this should be extended to Ramadan-focused diabetes education so that people can make informed decisions [3, 4]. In this study, only $45.6 \%$ of participants had access to diabetes education, of whom $45.2 \%$ received face-to-face educational sessions. Considering that Ramadan-focused education can enable individuals to reduce their risk of acute complications during fasts [3], the present study showed that this is an essential tool in patient management that is not yet being accessed by all patients. Healthcare professionals also seem to need more education on managing diabetes during Ramadan. One study in Pakistan found that almost one-third of general practitioners lacked knowledge of basic principles needed [11].

Of the 100 participants with type 1 diabetes who chose to fast, $63.0 \%$ reported that their reason was due to a personal decision, whereas $24.0 \%$ fasted to cope with pressure from their family and community. Of those people who did not fast, $60.0 \%$ reported a fear of diabetic complications as a reason for not fasting, and $50.0 \%$ had had previous experience of acute diabetic complications during fasting. Only $6.7 \%$ did not fast on the advice of their healthcare professional. Working conditions prompted $3.3 \%$ of participants to not fast during Ramadan.

The mean duration of fasting was 27 days with a daily duration of $16 \mathrm{~h}$; during this time, most people reported lifestyle changes. The majority of participants reported changes in working hours (67.5\%) and sleep duration $(67.2 \%)$, whereas changes in physical activity (45.0\%) and smoking (7.8\%) were less common. For those with changes in working hours, most $(84.3 \%)$ reported a decrease; however, changes were more balanced for those who reported a change in sleep duration, with $54.5 \%$ reporting a decrease and $45.5 \%$ reporting an increase. Food intake was also altered during Ramadan for $62.3 \%$ of participants. In those reporting changes, the proportions with increases and decreases were approximately equal for carbohydrates and sugars, whereas the majority of participants reported increases in protein $(92.6 \%)$ and vegetables $(91.8 \%)$ and decreases in fats $(60.5 \%)$. Of the $63.1 \%$ reporting changes in fluid intake, most $(80.5 \%)$ reported an increase. This change in food and fluid intake was accompanied by a change in insulin regimen in $84.6 \%$ of participants, and $72.1 \%$ modified their insulin dose.

It was anticipated that a greater proportion of participants (close to 100\%) would make a change to their insulin dose, but this was not observed in this study. In practice, $27.9 \%$ of participants did not change their insulin dose (regardless of type: rapid, short, intermediate, basal, or premix). Of these, $60.5 \%$ were on rapid-acting insulin. A high proportion of 
participants did not modify their insulin dose, which may have been due to inadequate glycemic control before Ramadan.

During Ramadan, not only do participants abstain from eating and drinking during daylight hours but they also drastically change aspects of their lifestyle and habits, which can affect glycemic control. In this study, there was a significant improvement in mean glycemic profile (based on HbA1c and FPG) after Ramadan fasting compared with before Ramadan. This occurred with no significant change in the incidence of confirmed hypoglycemia or number of confirmed hypoglycemic events during Ramadan compared with the previous 4 weeks. The lack of a significant increase in hypoglycemia in people with type 1 diabetes during Ramadan is clinically important as previous studies suggest an increase during Ramadan [5], and diabetes guidelines highlight the increased risk of hypoglycemia with fasting [3, 4].

In DAR-MENA participants with type 1 diabetes, lipid levels remained constant throughout Ramadan, except for total cholesterol, which was significantly increased $(P=0.021)$. In addition, there was no significant weight gain. While those who did not fast said that they were fearful of developing diabetic complications related to fasting, this did not appear to have happened in this study. Indeed, the incidence and number of AEs for severe hypoglycemia, confirmed hyperglycemia, and severe hyperglycemia did not change significantly during Ramadan compared with before. This is supported by a recent survey of 526 people with diabetes in Tunisia, of whom 51 had type 1 diabetes, who had fasted during Ramadan, which found that more than half of people had been treated with insulin and most were able to fast without interruption [12]. A recent literature review also found that Ramadan fasting in people with diabetes using insulin is feasible, with negligible major complications reported [13]. In DAR-MENA, diabetes education and the diabetic medication/dosing adjustments (primarily dose reductions) may have contributed to the low rates of hypoglycemia observed.

The results of the DAR-MENA type 1 diabetes analysis provide new up-to-date information on fasting during Ramadan, in comparison with previous studies, such as the EPIDIAR study, which had used the older insulins [5]. In EPIDIAR, fasting lasted for at least 15 days during Ramadan in $43 \%$ of people, and the mean number of fasting days was 23 days, which is shorter than the 27 days reported in this study [5]. A higher proportion of people with type 1 diabetes in the EPIDIAR study $(68 \%)$ received recommendations from their healthcare providers about fasting and diabetes during Ramadan than those who received education in DARMENA type 1 diabetes [5]. However, 57\% of participants had the same food intake as before, and $65 \%$ maintained their insulin dose during Ramadan [5]. The incidences of severe hypoglycemic episodes in participants with type 1 diabetes prior to Ramadan were similar in the DAR-MENA and EPIDIAR studies (0.05 vs 0.03 events/month/participant, respectively).

In EPIDIAR, the significant increase $(P=0.0174)$ in the mean \pm SD number of severe hypoglycemic episodes per month per person during Ramadan (0.14 \pm 0.6 events/ month) compared with the preceding year $(0.03 \pm 0.1$ events/month $)$ in the overall type 1 diabetes population may reflect the lack of dose change [5]. It should be noted that this significant increase roughly equates to only one more severe hypoglycemic event for each 5 months of fasting (+0.02 events/month/participant). Furthermore, the EPIDIAR study did not find a statistically significant increase in the mean \pm SD number of severe hypoglycemic episodes per month per person during Ramadan $(0.12 \pm 0.48$ events/month) compared with the preceding year $(0.02 \pm 0.05$ events/month $)$ in patients who fasted for at least 15 days [5].

New technologies for continuous glucose monitoring, such as glucose sensors and flash glucometers, can make fasting safer for people with type 1 diabetes who fast during Ramadan. Several studies have already shown the potential benefits of using an insulin pump during Ramadan [14]. In one study, those using an insulin pump performed self-monitoring of blood glucose more frequently than those on multiple daily insulin injections [7]. Unfortunately, the current high cost of an insulin pump restricts its use. 
Our analysis has some limitations. Firstly, the DAR-MENA study enrolled people with both type 1 and 2 diabetes, and did not require a specific number of each diabetes type. There is, therefore, a much lower number of participants with type 1 than type 2 diabetes. This is not unexpected as the proportion of the adult population with type 2 diabetes is higher than that with type 1 diabetes, but it does mean that this analysis is based on a small number of participants. Secondly, only those who believe that they are fit enough actually fast, and only a proportion of those are able to fast for the whole of the holy month. The results are, therefore, not representative of the entire type 1 diabetes population. While the analysis suggests that fasting during Ramadan is safe, this cannot be extrapolated to the whole of the type 1 diabetes population. Lastly, it is important to note that hypoglycemia, particularly symptomatic confirmed hypoglycemia, which was based on patient self-assessment, could potentially be under-reported as a result of impaired awareness of hypoglycemia or reluctance to measure SMPG levels. It is possible that participants perceived symptoms of hypoglycemia but did not assess SMPG levels; rather, they may have simply stopped activities and waited for symptoms to subside, as they may not have wanted to interrupt their fasting. Therefore, it was difficult to capture all hypoglycemic episodes and their severity. Ideally, the results of the current study would be validated in a future study based on continuous glucose monitoring.

\section{CONCLUSION}

This analysis of DAR-MENA provides new insight into the characteristics and pattern of fasting and care for people with type 1 diabetes before and during Ramadan. Despite the risks associated with fasting in this population, nearly half of participants fasted for the whole of the holy month. During Ramadan, mean glycemic control improved and there was no significant increase in confirmed and severe hypoglycemia events, which may have been due to pre-Ramadan diabetes education and modifications in diabetic dosing and regimen.
Although this study showed that confirmed or severe hypoglycemia and hyperglycemia during Ramadan have a similar incidence as during pre-Ramadan, the IDF-DAR Practical Guidelines recommend that people with type 1 diabetes should not fast, whether or not their diabetes is well controlled [3]. Since type 1 diabetes is associated with other problems such as hyperglycemia, hypovolemia, and diabetic ketoacidosis, those who insist on fasting need to work closely with their healthcare practitioner to ensure that they reduce their risk of complications.

\section{ACKNOWLEDGEMENTS}

The authors are grateful to all of the participants in the study. The authors would like to thank all contributors of the study, including the investigators from the 113 sites who participated in the data collection (Table S8 in the supplementary material), and the analysts, Soha Ali (Senior Biostatistician, DATACLin CRO) and Mohamad Taha (Biostatistics Manager, DATACLin CRO), for providing the statistical and analytical execution of this study. The authors would also like to acknowledge Helena Andersson, Sanofi, for the coordination of the development of this manuscript.

Funding. Sponsorship for this study was provided by Sanofi. This study was conducted in collaboration with DAR International Alliance. Sanofi provided financial support for the conduct of the study research. All authors had full access to all of the data in this study and take complete responsibility for the integrity of the data and accuracy of the data analysis. Sanofi was involved in the study design, collection, analysis and interpretation of the data, as well as data checking of information provided in the manuscript. However, ultimate responsibility for opinions, conclusions, and data interpretation lies with the authors. The journal's Rapid Service and Open Access Fees were also funded by Sanofi. 
Medical Writing and Editorial Assistance. Editorial assistance in the preparation of this article was provided by Breanne Landry and Debby Moss of Caudex (Oxford, UK). Support for this assistance was funded by Sanofi.

Authorship. All named authors meet the International Committee of Medical Journal Editors (ICMJE) criteria for authorship for this article, take responsibility for the integrity of the work as a whole, and have given their approval for this version to be published.

Authorship Contributions. Mohamed M. Hassanein and Khier Djaballah developed the study concept and design. All authors contributed to the data analysis or interpretation of the results, and critically revised, provided final approvals of, and are accountable for the accuracy and integrity of the manuscript. Fatheya F. $\mathrm{Al}$ Awadi and Khier Djaballah are the guarantors of this work and, as such, had full access to all the data in the study and take responsibility for the integrity of the data and the accuracy of the data analysis.

Disclosures. Fatheya F. Al Awadi, Sobia Sabir Ali, Abdulrahman Al Shaikh, Mohamad Omar Abu-Hijleh, and Faris Abdul Kareem Khazaal have nothing to disclose. Akram Echtay: Advisory board member for AstraZeneca, Boehringer Ingelheim, Merck, and Novo Nordisk; speaker for AstraZeneca, Boehringer Ingelheim, Eli Lilly, Merck, Novartis, Novo Nordisk, and Sanofi. Monira Al Arouj: Advisory board member for Merck and Sanofi. Naim Shehadeh: Advisory board member for Boehringer Ingelheim, Merck, Novo Nordisk, and Sanofi; consultant for Merck, Novo Nordisk, and Sanofi; recipient of grant from Merck, Novo Nordisk, and Sanofi; research investigator for AstraZeneca, Merck, Novo Nordisk, and Sanofi; speaker for Boehringer Ingelheim, Eli Lilly, Novo Nordisk, and Sanofi; stock owner from Novo Nordisk. Khier Djaballah: Employee of Sanofi. Cecile DessaptBaradez: Employee and shareholder of Sanofi. Abdullah Bennakhi: Advisory board member for Merck and Sanofi. Mohamed El Hassan Gharbi: Advisory board member for Janssen and Servier; research investigator for Sanofi; speaker for
Merck, Novartis, Novo Nordisk, Sanofi, and Servier. Khaled El Sayed El Hadidy: Advisory board member for Merck and Sanofi; speaker for AstraZeneca, Eli Lilly, Merck, and Sanofi. Mohamed M. Hassanein: Advisory board member for Boehringer Ingelheim, Novo Nordisk, and Sanofi; speaker for Eli Lilly, Janssen, LifeScan BI, Merck, Novo Nordisk, and Sanofi.

Compliance with Ethics Guidelines. The manuscript followed the STrengthening the Reporting of OBservational studies in Epidemiology (STROBE) guidelines [10]. DAR-MENA was conducted in accordance with the ethical principles laid down in the Declaration of Helsinki and complied with all international guidelines, as well as national laws and regulations of each country in which the registry study was performed. The study protocol was approved by the ethics committee of each participating center (please see Table $\$ 9$ in the supplementary material for ethics committee names). Participants signed a consent form to participate in the study. Please note that this study was not added to a clinical trial registry as it was a noninterventional, prospective, observational study that did not meet the definition for clinical trial registration as specified by the World Health Organization.

Data Availability. Qualified researchers may request access to participant-level data and related study documents, including the clinical study report, study protocol with any amendments, blank case report form, statistical analysis plan, and dataset specifications. Participantlevel data will be anonymized, and study documents will be redacted to protect the privacy of trial participants. Further details on Sanofi's data sharing criteria, eligible studies, and process for requesting access can be found at https://www.clinicalstudydatarequest.com.

Open Access. This article is licensed under a Creative Commons Attribution-NonCommercial 4.0 International License, which permits any non-commercial use, sharing, adaptation, distribution and reproduction in any medium or format, as long as you give appropriate credit to the original author(s) and the source, provide 
a link to the Creative Commons licence, and indicate if changes were made. The images or other third party material in this article are included in the article's Creative Commons licence, unless indicated otherwise in a credit line to the material. If material is not included in the article's Creative Commons licence and your intended use is not permitted by statutory regulation or exceeds the permitted use, you will need to obtain permission directly from the copyright holder. To view a copy of this licence, visit http://creativecommons.org/licenses/by$\mathrm{nc} / 4.0 /$.

\section{REFERENCES}

1. Qur'an. Surah al-Baqarah. 2:185.

2. Al-Arouj M, Bouguerra R, Buse J, et al. Recommendations for management of diabetes during Ramadan. Diabetes Care. 2005;28:2305-11.

3. Hassanein M, Al-Arouj M, Hamdy O, et al. Diabetes and Ramadan: practical guidelines. Diabetes Res Clin Pract. 2017;126:303-16.

4. Al-Arouj M, Assaad-Khalil S, Buse J, et al. Recommendations for management of diabetes during Ramadan: update 2010. Diabetes Care. 2010;33: 1895-902.

5. Salti I, Benard E, Detournay B, et al. A populationbased study of diabetes and its characteristics during the fasting month of Ramadan in 13 countries: results of the epidemiology of diabetes and Ramadan 1422/2001 (EPIDIAR) study. Diabetes Care. 2004;27:2306-11.

6. Hassanein M, Al Awadi FF, El Hadidy KES, et al. The characteristics and pattern of care for the type 2 diabetes mellitus population in the MENA region during Ramadan: an international prospective study (DAR-MENA T2DM). Diabetes Res Clin Pract. 2019;151:275-84.

7. Alamoudi R, Alsubaiee M, Alqarni A, et al. Attitudes and habits of patients with type 1 diabetes during fasting Ramadan. J Clin Transl Endocrinol. 2018;14: $1-4$.

8. Eid YM, Sahmoud SI, Abdelsalam MM, Eichorst B. Empowerment-based diabetes self-management education to maintain glycemic targets during Ramadan fasting in people with diabetes who are on conventional insulin: a feasibility study. Diabetes Spectr. 2017;30:36-42.

9. Reiter J, Wexler ID, Shehadeh N, Tzur A, Zangen D. Type 1 diabetes and prolonged fasting. Diabet Med. 2007;24:436-9.

10. STROBE. STrengthening the Reporting of OBservational studies in Epidemiology. 2014. https://www. strobe-statement.org/index.php?id=strobe-home. Accessed Jul 2018.

11. Ahmedani MY, Hashmi BZ, Ulhaque MS. Ramadan and diabetes-knowledge, attitude and practices of general practitioners; a cross-sectional study. Pak J Med Sci. 2016;32:846-50.

12. Abid M, Hsairi M, Elleuch M, Ben Aissa E. Survey on diabetic patients treated with insulin during the fasting month of Ramadan. Int J Gen Med. 2018;11: $33-40$.

13. Alabbood MH, Ho KW, Simons MR. The effect of Ramadan fasting on glycaemic control in insulin dependent diabetic patients: a literature review. Diabetes Metab Syndr. 2017;11:83-7.

14. Ahmed MH, Husain NE, Elmadhoun WM, Noor SK, Khalil AA, Almobarak AO. Diabetes and Ramadan: a concise and practical update. J Family Med Prim Care. 2017;6:11-8. 